\title{
ATITUDE E MOTIVAÇÃO EM RELAÇÃO AO \\ DESEMPENHO ACADÊMICO DE ALUNOS DO CURSO DE \\ GRADUAÇÃO EM ADMINISTRAÇÃO EM DISCIPLINAS DE \\ ESTATÍSTICA: FORMAÇÃO DE CLUSTERS
}

\author{
ATIITUDE AND MOTIVATION IN RELATION TO \\ THE ACADEMIC PERFORMANCE OF UNDERGRADUATE \\ STUDENTS FROM STATISTICS COURSES IN \\ BUSINESS ADMINISTRATION: THE FORMATION OF CLUSTERS
}

\section{Recebido em: 27/06/2012 Aprovado em: 03/08/2012 Avaliado pelo sistema double blind review Editora Científica: Manolita Correia Lima}

\section{GUSTAVO SALOMÃOVIANA salomao@fearp.usp.br FACULDADE SÃO LUÍS DE JABOTICABAL}

\author{
ADRIANA BACKX NORONHA VIANA \\ UNIVERSIDADE SÃO PAULO
}

\begin{abstract}
RESUMO
O objetivo do presente artigo é analisar a formação de clusters, levando-se em consideração: variáveis de caracterização do aluno, atitude perante a Estatística, motivação acadêmica e desempenho. Os dados foram coletados por meio da aplicação de um questionário a 278 estudantes do curso de graduação em Administração, em duas faculdades públicas do estado de São Paulo, no segundo semestre de 20Ir. O questionário foi composto de perguntas para a caracterização dos alunos, Escala de Motivação Acadêmica (EMA), Escala de Atitude perante a Estatística (SATS), bem como uma nota relativa à autopercepção de aprendizado, sendo que a nota na disciplina foi obtida junto ao docente responsável. Verificou-se que apenas a área de interesse mostrou-se como possível variável diferenciadora, dada a composição dos 3 grupos. O grupo de alunos com maior interesse na área de Finanças apresentou as maiores pontuações tanto em relação à atitude perante a Estatística como em relação à motivação acadêmica, exceto Desmotivação, apresentando também, os maiores valores para o desempenho tanto em relação à nota quanto em relação à autopercepção.

Palavras-chave: atitude perante a estatística; motivação acadêmica; teoria da autodeterminação; desempenho acadêmico.
\end{abstract}

\section{ABSTRACT}

The aim of this paper is to analyze the formation of clusters, taking into consideration: the characterization variables of students and their attitude to statistics, academic motivation and performance. Data was collected using a questionnaire answered by 278 undergraduate Business Administration students from two public colleges in the state of São Paulo, in the second half of 2011. The questionnaire consisted of questions to characterize the students, the Academic Motivation Scale (AMS), the Survey on Attitudes Towards Statistics (SATS), as well as a grade on the perception of learning, with the grade for the discipline obtained from the respective professor. It was found that only the area of interest proved to be a possible differentiating variable given the composition of the three groups. The largest group of students with interest in the area of Finance presented the highest scores, both in terms of their attitude towards statistics as in relation to academic motivation, with the exception of demotivation. This group also presented the highest scores for performance both in relation to the grade, as well as in relation to their self-perception.

Keywords: attitude toward statistics; academic motivation; self-determination theory; academic performance. 


\section{INTRODUÇÃO}

Em uma sociedade que apresenta ênfase no conhecimento, torna-se importante analisar a quantidade significativa de informações contidas nos bancos de dados, objetivando transformá-las em conhecimentos utilizáveis, tanto para fins comerciais, quanto científicos (ARA, 2006). O crescimento na demanda para a análise de dados pode ser atribuída, em grande parte, ao exponencial aumento do poder computacional e da capacidade de coleta de dados (BROwN; KASS, 2009).

Neste sentido a Estatística voltada para a Administração compreende um conjunto de técnicas que auxiliam o administrador no processo de tomada de decisão, reduzindo, portanto, a incerteza que é inerente ao processo decisório (MILAGRE, 200I).

Burand (2009, p.I4) afirma que a Estatística fornece uma chave para a Administração inteligente, bem como para a vantagem competitiva. Desse modo, o conhecimento do conteúdo de estatística pelo administrador poderá ser aplicado tanto na esfera da administração privada, como da pública. $\mathrm{Na}$ área empresarial, as aplicações mais comuns são nas áreas de marketing, finanças, pesquisa operacional, produção e recursos humanos (PIMENTEL, 2009).

Pensando, portanto, na atuação do aluno no mercado, vislumbram-se, na área de Estatística, diversas possibilidades. A necessidade da ciência, da tecnologia, dos negócios e do governo por estatística é enorme e cresce rapidamente, proporcionando uma carência de profissionais nesta área (BROWN; KASS, 2009).

A formação Estatística do aluno de graduação em Administração tornase de fundamental importância para a construção de um profissional que atenda às novas demandas. No entanto, segundo Nolan e Speed (I999, p.oI), os alunos apresentam dificuldades na integração entre os conteúdos de Estatística e as demais áreas do curso, bem como em aplicarem, de maneira autônoma, o conteúdo em um determinado projeto acadêmico ou no exercício profissional. 
O desafio do processo de ensino-aprendizagem de Estatística, tanto para os professores, quanto para os alunos do curso de graduação em Administração, é um aspecto importante a ser considerado (MANTOVANI, 2008).

Mas quais fatores podem influenciar no desempenho acadêmico do aluno? Singh, Granville e Dika (2002, p.323) asseveram que há um relacionamento positivo entre a motivação acadêmica e a atitude do aluno em relação ao seu desempenho acadêmico.

Este trabalho apresenta como objetivo analisar a formação de grupos por meio das seguintes variáveis: variáveis de caracterização do aluno, dimensões da atitude em relação à Estatística, dimensões da motivação acadêmica, nota na disciplina de Estatística e autopercepção de desempenho na disciplina.

A presente pesquisa justifica-se pela necessidade de aperfeiçoamento do ensino do conteúdo de estatística para o curso de Administração, tendo em vista sua importância e vasta aplicabilidade no exercício da atividade do administrador. É importante destacar, ainda, que tais implicações encontram-se presentes tanto na práxis do profissional da Administração pública e privada, quanto na atividade de pesquisa na área da Administração.

O presente estudo vai ao encontro, tanto dos interesses dos docentes da área de Estatística aplicada à Administração, quanto dos docentes das outras disciplinas do curso, uma vez que propiciará, diretamente, a maior compreensão da interação das variáveis de caracterização, das dimensões motivacionais, atitudinais perante a Estatística e do desempenho. Portanto, tal resultado contribui para o desenvolvimento do ensino de Estatística, uma vez que permite a identificação de possíveis padrões de grupos, que envolve caracterização, atitude, motivação e desempenho, transformandose em um importante subsídio para o docente de Estatística refletir sobre o ensino e o aprendizado de Estatística 


\section{REFERENCIAL TEÓRICO}

Para o desenvolvimento do estudo, consideraram-se no referencial teórico estudos referentes à relação entre Administração e Estatística, o ensino de Estatística para administração, a motivação acadêmica e a atitude perante a Estatística, conforme descritos a seguir.

\section{RELAÇÃO ENTRE ADMINISTRAÇÃO E ESTATÍSTICA}

Maximiano (2004, p.26) define Administração como o processo de tomar e colocar em prática decisões acerca de objetivos e utilização de recursos, abrangendo quatro tipos principais de decisões, também chamadas de processos e funções: planejamento (definição de objetivos e recursos), organização (disposição de recursos numa determinada estrutura), execução (realização dos planos) e controle (verificação dos resultados).

De maneira geral, o processo de Administração envolve, basicamente, o processo reiterado de tomada de decisões, como assevera Simon (1960 apud PAIVA, 2002, p. Io): "tomar decisões e administrar são sinônimos".

Como as decisões relativas à Administração referem-se a recursos, tanto humanos, quanto produtivos escassos, faz-se necessário o desenvolvimento de um processo decisório que considere tais limitações (BASTOS; OLIVEIRA; OLIVEIRA, I998).

Neste sentido, surge a Estatística como uma importante área para a Administração, tendo em vista que é esperado que profissionais dessa área tenham habilidades em análise de dados, sendo um importante componente do processo de tomada de decisão (SIRIAS, 2002). Mais do que simplesmente o processo de tomada de decisão, faz-se necessário um processo ágil de tomada de decisões seguras, presente cotidianamente na atividade do administrador, baseando-se em informações por trás de fatos / dados e não simplesmente em opiniões (MILAGRE, 200I).

Para Gould (2004, p.7), a variabilidade é o elemento que torna as decisões em face da incerteza tão difíceis. Dessa maneira, a variabilidade é que transforma a estatística em algo tão desafiador e interessante, permitindo interpretar, modelar e fazer previsões de dados. 
A Estatística aplicada à Administração é utilizada, portanto, como uma verdadeira colaboradora do processo de tomada de decisão, nos mais diversos níveis hierárquicos. Dessa forma, a Estatística torna mais objetivo o processo de tomada de decisão, diminuindo a incerteza relacionada à escolha de determinada alternativa (MANTOVANI; viANA, 2007).

\section{O ENSINO DE ESTATÍSTICA PARA A ADMINISTRAÇÃO}

A crescente utilização da Estatística pela Administração torna obrigatória a abordagem de tal conteúdo pelas faculdades de Administração. Assim, as escolas de negócio, quase mundialmente, incluem o conteúdo de estatística em seus currículos de formação; no entanto, tal conteúdo tem provocado ampla insatisfação (MCALEVEY; SULLIVAN, 200I).

No entanto, estudantes que cursam boas disciplinas de estatística acabam perdendo o medo e, de fato, começam a achar o assunto interessante, não somente para suas carreiras, mas passam a ver o próprio mundo de uma maneira diferente (WOOLFORD; CLEARY, 20IO).

No ano de 1965, com o artigo intitulado The Teaching of Statistics in Business Schools, já se destacava a preocupação no ensino de estatística voltado à área de negócios, uma vez que tal artigo já reconhecia o grande número de aplicabilidades que o conteúdo de Estatística poderia oferecer para a resolução de problemas organizacionais, bem como já abordava algumas preocupações com o ensino, como, por exemplo, em relação à formação dos docentes para ministrarem aulas em disciplinas de estatística aplicada à área de negócios (COx, 1965).

Segundo Dargahi-Noubary e Growney (1998), disciplinas introdutórias de Estatística sempre se apresentaram como um desafio para os professores, com o desenvolvimento de uma extensa pesquisa para tentar torná-las mais fáceis de serem ensinadas e de serem compreendidas.

Um aspecto importante, relatado por Meng (2009), refere-se às grandes implicações de uma disciplina introdutória de estatística. O autor destaca que uma formação estatística de baixa qualidade na área de Administração poderá ocasionar profundos e prolongados efeitos, como, por exemplo, a 
utilização de conceitos e ferramentas de maneira equivocada, tanto na área de pesquisas acadêmicas quanto na práxis da Administração.

Como salientam Woolford e Cleary (2010), se a área de estatística sofresse algumas adaptações para a educação na área de negócios, representaria um importante passo na geração de profissionais mais efetivos, tanto na academia, quanto nos negócios.

Há, portanto, a necessidade de empreender grandes esforços para promover a mudança da ideia de que "Statistics is easy to teach, but hard (and boring) to learn" para uma concepção de "Statistics is hard to teach, but easy (and fun) to learn". Cursos especificamente projetados, cuidadosamente preparados e bem ensinados apresentam maior chance de convencer os alunos de que estatística é um assunto de fundamental importância na formação, especialmente, para alunos que não têm como objetivo a carreira estatística (MENG, 2009).

Surgem, portanto, como temáticas de grande importância no ensino de tal conteúdo aplicado à Administração, tanto a motivação acadêmica dos alunos como a atitude dos alunos perante o conteúdo de Estatística.

\section{MOTIVAÇÃO ACADÊMICA}

São inúmeras as tentativas de conceituação do termo motivação, havendo grande dificuldade para a construção de um significado unânime. Segundo uma perspectiva ampla encontrada na literatura, é possível defini-la como um conjunto de determinantes ambientais, forças internas e incentivos, que impelem o organismo a executar uma determinada tarefa (WITTER; LOMÔNACO, 1984).

Corroborando tal afirmativa, Smith, Davy e Rosenber (2010, p.323) e Hegarty (2010, p.48) afirmam que, mesmo depois de décadas, os pesquisadores ainda apresentam grande interesse na relação entre motivação e resultados educacionais.

A motivação no contexto acadêmico é considerada como um determinante crítico do nível e da qualidade da aprendizagem e do desempenho dos alunos. Dessa forma, um estudante motivado apresenta-se ativamente envolvido no 
processo de ensino-aprendizagem, engajando-se em atividades desafiadoras, utilizando estratégias adequadas e buscando desenvolver novas habilidades de compreensão e de domínio (GUIMARÃes; BUROCHOvit, 2004).

Entre as várias abordagens sobre motivação acadêmica, destaca-se a teoria da autodeterminação motivacional, que procura analisar como as tendências naturais para o crescimento e o atendimento das necessidades psicológicas interagem com as condições sociais, que podem alimentar ou frustrar essas fontes naturais (REEVE; DECI; RYAN, 2004).

De acordo com essa teoria, a motivação não deve ser vislumbrada numa perspectiva unidimensional. Como proposta para a compreensão da motivação humana, propõe-se a existência sim de três dimensões: motivação intrínseca, motivação extrínseca e desmotivação (DECI; RYAN, 1985).

Segue abaixo um conjunto de definições de Cavenaghi (2009), acerca dos elementos componentes do continuum da motivação, segundo a teoria da autodeterminação.

A desmotivação pode ser caracterizada como um estado no qual falta ao estudante a intencionalidade de agir, podendo resultar da desvalorização da atividade, do sentimento de falta de competência para realizá-la ou porque crê que a atividade não trará os resultados almejados.

A regulação externa é o tipo de motivação extrínseca menos autodeterminada, tendo em vista que o comportamento é controlado por contingências externas. É o tipo de motivação que faz com que o aluno se envolva em determinada atividade para obter nota ou para não ficar sem nota.

A regulação introjetada é um tipo de motivação extrínseca que envolve a ação por uma regulação internalizada, porém não pessoalmente aceita. É o tipo de motivação que faz com que o aluno realize determinada atividade para afirmar seu autovalor ou para amenizar uma ameaça.

A regulação integrada é o tipo de motivação extrínseca mais autodeterminada, no sentido de que, além de realizar a atividade por saber de sua importância na aprendizagem, o aluno a integra dentro de si e a aceita como sua. 
Por motivação intrínseca entende-se a motivação inata, sendo aquela que se origina de maneira espontânea das necessidades psicológicas e se manifesta pelo interesse na atividade em si.

Segue a Figura I contendo o continuum de autodeterminação proposto por Deci e Ryan (2000), iniciando-se na desmotivação, passando para a regulação externa, regulação introjetada, regulação identificada, regulação integrada e motivação intrínseca, indo, portanto, de uma ausência total de autodeterminação até a autodeterminação plena.

Figura 1 Continuum da autodeterminação motivacional

\begin{tabular}{|c|c|c|c|c|c|c|}
\hline \multirow{2}{*}{ 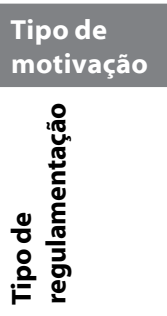 } & \multirow{2}{*}{ 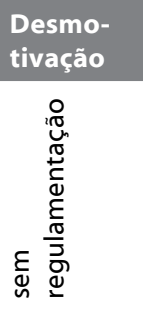 } & \multicolumn{4}{|c|}{ Motivação extrínseca } & \multirow{2}{*}{ 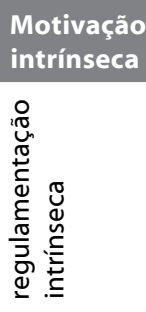 } \\
\hline & & 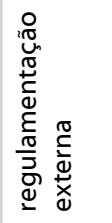 & 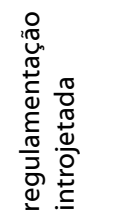 & 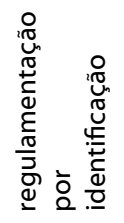 & 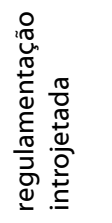 & \\
\hline $\begin{array}{l}\text { Locus da } \\
\text { casualidade }\end{array}$ & impessoal & externa & $\begin{array}{l}\text { pouco } \\
\text { externa }\end{array}$ & $\begin{array}{l}\text { pouco } \\
\text { interna }\end{array}$ & interno & interno \\
\hline
\end{tabular}

Fonte: Deci e Ryan (2000, p.237).

A Echelle de Motivation en Education (EME) surgiu no de 1989, constituída por 28 itens, subdivididos em três dimensões: motivação intrínseca, motivação extrínseca e desmotivação (vallerand et al., 1989).

As três tipologias de motivação intrínseca podem ser identificadas como: motivação intrínseca para saber, motivação intrínseca para realizar coisas e motivação intrínseca para vivenciar estímulos.

Apresenta-se uma breve descrição dos três componentes da motivação intrínseca, de acordo com o criador da escala:

a. Motivação intrínseca para saber: fazer algo pelo prazer e satisfação que decorre de aprender, explorar ou entender coisas novas. Por exemplo, estudantes que são intrinsecamente motivados para saber, leem um livro simplesmente pelo prazer de aprender algo novo (VALLERAND et al., 1992). 
b. Motivação intrínseca para realização: fazer algo pelo prazer e satisfação que decorre da busca de realização ou criação de coisas. Por exemplo, estudantes que estendem seu trabalho além do pedido, tendo em vista a satisfação de superação (valleRAND ET al., 1992).

c. Motivação intrínseca para vivenciar estímulos: fazer algo a fim de experimentar sensações estimulantes, de natureza sensorial ou estética. Por exemplo, estudantes que vão à aula tendo em vista a estimulação proporcionada por discussões realizadas ou que lêem um livro pelo sentimento de prazer cognitivo de algumas passagens (VALLERAND et al., 1992).

Contrariamente à motivação intrínseca, a motivação extrínseca pertence a um conjunto de comportamentos em que a realização de determinada atividade decorre de um fator externo ao indivíduo ou, segundo Sobral (2003), faz-se algo devido à consequência ou ao desfecho da ação.

Propôs-se a existência de três tipos de motivação extrínseca, que podem ser ordenadas ao longo de um continuum da autodeterminação. Do menor para o maior nível de autodeterminação, verifica-se: regulação externa, regulação por introjeção e regulação por identificação.

Segue abaixo maior pormenorização dos três componentes da motivação extrínseca, de acordo com o criador da escala:

a. Regulação externa: fazer algo porque se sente pressionado por outros a fazê-lo. Como exemplo, verifica-se o seguinte: Um estudante que estuda na noite anterior à realização de um exame porque os pais o obrigam (VALLERAND et al., 1992).

b. Regulação por introjeção: fazer algo porque se pressiona a si próprio a fazê-lo. Como exemplo, verifica-se o seguinte: Um estudante que estuda na noite anterior à realização de um exame porque acredita que os bons estudantes fazem isso (vallerand et al., 1992).

c. Regulação por identificação: fazer algo porque se decidiu fazê-lo. Como exemplo, verifica-se o seguinte: Um estudante que estuda à noite porque acredita que é algo importante para ele (vallerand et al., 1992). 
Existe ainda um terceiro tipo de construto motivacional importante para compreender o comportamento humano: a desmotivação. Indivíduos desmotivados são aqueles que não percebem as contingências entre os resultados e suas ações. Eles não são intrínseca, nem extrinsecamente motivados (DECI; RYAN, 1985).

\section{ATITUDE PERANTE A ESTATÍSTICA}

Segundo Vendramini, Silva e Dias (2009), considera-se a atitude em relação à Estatística como uma resposta afetiva dada por um determinado indivíduo, em face de uma situação em que este utilizará o referido conteúdo, seja cursando uma disciplina, seja analisando os dados de uma pesquisa.

Em um clássico artigo, Wise (1985 relata que atitudes negativas caracterizam-se como obstáculos para a aprendizagem do aluno, devendo, portanto, tornar-se um dos objetivos dos docentes desta área a promoção do desenvolvimento de atitudes positivas nos alunos.

Torna-se tarefa de grande importância o desenvolvimento do conhecimento acerca da atitude dos alunos perante a estatística, tendo em vista que possíveis modificações podem alterar o desempenho desses estudantes na referida área. Como salientam Gal, Ginsburg e Schau (1997), as atitudes e crenças dos alunos em relação à estatística podem impedir ou auxiliar a aprendizagem, influindo na forma como será desenvolvido o pensamento estatístico nestes alunos, bem como na aplicação de tal conteúdo fora da sala de aula.

Para Schau et al. (1995), a atitude perante a estatística comporta-se de maneira multidimensional, sendo composta da dimensão afetividade, competência cognitiva, valor e dificuldade. É importante, portanto, considerar a definição de cada dimensão, segundo o desenvolvedor da escala (scHAu et al., 1995):

a) Afetividade: sentimentos positivos e negativos em relação à estatística;

b) Competência cognitiva: atitudes em relação ao conhecimento intelectual e às habilidades exigidas pela Estatística;

c) Valor: atitudes em relação à utilidade, relevância e valor da Estatística na vida pessoal e profissional do indivíduo; 
ATITUDE E MOTIVAÇÃO EM RELAÇÃO AO DESEMPENHO ACADÊMICO DE ALUNOS DO CURSO DE GRADUAÇÃO EM ADMINISTRAÇÃO EM DISCIPLINAS DE ESTATÍSTICA: FORMAÇÃO DE CLUSTERS

d) Dificuldade: atitudes em relação à dificuldade da estatística como assunto. Schau et al. (1995) elaboraram, para a mensuração de tais dimensões, a escala Survey of Attitudes Toward Statistics (SATS) composta de 28 afirmações, contendo atitudes positivas e negativas em relação à Estatística, afirmações que, agrupadas, formam as dimensões da atitude elencadas acima. 


\section{MÉTODO}

A natureza do método adotado na presente pesquisa classifica-se como quantitativa, tendo em vista que, segundo Oliveira (1999), a pesquisa quantitativa significa quantificar opiniões e dados, utilizando como recursos de análise técnicas estatísticas desde as mais simples técnicas de estatística descritiva até as ferramentas mais avançadas de estatística inferencial.

Os dados foram coletados por meio da aplicação de um questionário, que contém elementos para a caracterização dos alunos, escala denominada de Escala de Motivação Acadêmica (EMA), Escala de Atitude perante a Estatística (SATS), além da nota obtida pelo aluno na disciplina, bem como uma nota atribuída pelo próprio aluno ao seu desempenho em Estatística.

A população que serviu de objeto de análise para a presente pesquisa foi composta de 455 alunos, que cursaram disciplinas obrigatórias de Estatística no curso de graduação em Administração, em duas faculdades públicas brasileiras. Destaca-se que os respondentes encontraram-se compreendidos entre o segundo e o quarto semestre. Os questionários foram aplicados no segundo semestre do ano de 201 .

Foi obtida uma amostra não probabilística composta de 289 alunos, número este que representa cerca de $60 \%$ da população, servindo, portanto, como considerável amostra para a realização das análises.

Observou-se a participação de 67,99\% da Ir e 32,0I\% da I2. Os questionários foram aplicados em disciplinas de turmas do período diurno e noturno observando-se a participação de 34,6\% de alunos do curso diurno e $65,4 \%$ de alunos do curso noturno. A Tabela I apresenta a distribuição de frequência dos questionários por IEs e período do curso.

Tabela 1 Distribuição dos questionários por IEs e período do curso

\begin{tabular}{|llll|}
\hline IES $\backslash$ Período do curso & Diurno & Noturno & Total \\
\hline 11 & 83 & 106 & 189 \\
\hline 12 & 13 & 76 & 89 \\
\hline Total & $\mathbf{9 6}$ & $\mathbf{1 8 2}$ & $\mathbf{2 7 8}$ \\
\hline
\end{tabular}


Em relação às disciplinas em que foram coletados os dados, observou-se uma participação de alunos respondentes, comparativamente ao número de alunos matriculados, indo desde $13 \%$ até $97,8 \%$, conforme apresentado na Tabela 2.

Tabela 2 Distribuição dos questionários por disciplina (\%)

\begin{tabular}{|lllll|}
\hline Instituiçã॰o & Disciplina & $\begin{array}{l}\text { No de alunos } \\
\text { matriculados }\end{array}$ & $\begin{array}{l}\mathbf{N}^{\circ} \text { de } \\
\text { questionários } \\
\text { respondidos }\end{array}$ & $\begin{array}{l}\text { No de } \\
\text { questionários } \\
\text { utilizados } \\
\text { (\% }\end{array}$ \\
matriculados)
\end{tabular}

Em relação à distribuição, no que concerne à etapa do curso em que o aluno se encontrava matriculado, observou-se uma participação de 85 (31\%) de alunos do segundo semestre), I54 (55\%) do terceiro semestre) e 39 (I4\%) de alunos do quarto semestre).

A opção pela escolha das faculdades deu-se em função das características comuns compartilhadas pelos dois cursos.

O ato de responder o questionário foi completamente voluntário por parte dos alunos, o que não possibilitou a utilização da população, mas sim, de uma amostra dela. Porém, buscou-se o maior número de respondentes possível, por meio do oferecimento de explicações sobre a pesquisa, 
bem como de sua importância na formação do profissional da área de Administração.

De acordo com várias pesquisas já realizadas, na mesma seara, fazse importante considerar as variáveis de caracterização dos alunos na interação com a atitude perante a Estatística, Carlson e Winquist (20II), Devaney (2010) e Pimentel (2009); assim como em relação à motivação acadêmica, Smith, Davy e Rosenber (2010), Brouse et al. (2010), Davis (2009), Barkoukis et al (2008) e Lavender (2005). Como variáveis de caracterização foram consideradas:

I. Sexo (masculino / feminino);

II. Idade (idade do aluno na data de resposta do questionário);

III. Atividade desempenhada atualmente (estuda somente / realiza estágio e estuda / realiza iniciação científica e estuda / trabalha meio período e estuda / trabalha em período integral e estuda / outra);

Iv. Área de maior interesse na Administração (finanças / marketing / recursos humanos / produção / outra)

Para a mensuração da motivação acadêmica dos alunos foi utilizada a Escala de Motivação Acadêmica (EMA), em sua versão portuguesa, adaptada por Sobral (2003). A escolha da referida escala se deu em função de sua característica multidimensional, indo ao encontro da Teoria da Autodeterminação.

A Escala de Motivação Acadêmica (EMA) é composta por 28 afirmações, utilizando-se deuma escala de 7 pontos de Likert (nenhuma correspondência $=\mathrm{I}$, moderada correspondência $=4$ e total correspondência $=7$ ).

Para a mensuração da atitude dos alunos perante a Estatística, foi utilizada a escala Survey of Attitudes Toward Statistics (SATS), instrumento já validado para a língua portuguesa de acordo com Vendramini et al. (20II) . A escolha da referida escala se deu em decorrência de sua característica de mutidimensionalidade, tendo em vista que, de acordo com esta escala, a atitude do aluno perante a Estatística é composta por quatro dimensões: afetividade, competência cognitiva, valor e dificuldade. 
A Escala de Atitude perante a Estatística compõe-se de 28 afirmações, que contêm atitudes positivas e negativas em relação à Estatística, afirmações que, agrupadas, formam as dimensões da atitude elencadas acima. O referido instrumento utiliza-se de uma escala de 7 pontos de Likert ( $\mathrm{I}=$ discordo fortemente, $4=$ nem discordo nem concordo, $7=$ concordo fortemente), sendo que, quanto maior a pontuação, mais positiva será a atitude perante a estatística (schau et al., i995).

Para mensuração do desempenho acadêmico, duas possibilidades foram consideradas: a média final atribuída ao aluno na disciplina de Estatística aplicada à Administração e a autopercepção do aluno em relação ao seu próprio desempenho. Destaca-se que a última variável (autopercepção do desempenho) foi também relacionada com a atitude do aluno em relação à Estatística no estudo de Vendramini, Silva e Dias (2009).

Uma importante consideração deve ser feita em relação à forma de mensuração do desempenho do aluno. Neste sentido, optou-se pela média obtida no final da disciplina, mesmo levando-se em consideração possíveis distorções decorrentes da realização de trabalhos.

Em relação à análise dos dados, segundo Hair et al. (2005, p. 38I), acadêmicos e pesquisadores frequentemente encontram situações mais bem resolvidas pela definição de grupos de objetos homogêneos, sejam eles indivíduos, empresas, produtos ou mesmo comportamentos.

Neste sentido, a técnica mais comumente utilizada para esta finalidade é a denominada análise de cluster, possibilitando reunir indivíduos em grupos cujos objetos são mais parecidos uns com os outros do que com os indivíduos de outros grupos (HAIR et al., 2005).

Desse modo buscou-se a formação de clusters, considerando o desempenho, mensurado como a nota obtida na disciplina de Estatística, autopercepção de desempenho do aluno, as dimensões motivacionais e atitudinais, considerando ainda as variáveis de caracterização. 


\section{RESULTADOS E ANÁLISES}

A análise de cluster foi realizada sobre as variáveis da escala sATs (Afeto, Competência cognitiva, Valor e Dificuldade), da escala EMA (Motivação intrínseca para saber, Motivação intrínseca para realização, Motivação intrínseca para vivenciar estímulos, Motivação extrínseca - identificação, Motivação extrínseca - introjeção, Motivação extrínseca - controle externo e Desmotivação), nota na disciplina e autopercepção de desempenho.

Utilizou-se o método hierárquico com processo de agrupamento Ward's e distância euclidiana quadrada como medida de distância para a realização da análise.

Por meio da visualização inicial e do dendograma foi possível a formação de três grupos, sendo que $38 \%$ das pessoas (I06 pessoas) foram agrupadas no Grupo I; 31\% (86 pessoas) foram agrupadas no Grupo 2; e 31\% (86 pessoas) foram agrupadas no Grupo 3.

Uma importante consideração deve-se à caracterização dos grupos. Neste sentido, primeiro fez-se uma análise descritiva das variáveis de caracterização, para que, em um segundo momento, fossem analisadas as diferenças, estatisticamente significantes, entre os grupos.

No que concerne ao gênero dos grupos formados, observou-se uma superior participação masculina em todos os grupos, indo desde 54,7\% (Grupo 2) até 72,6\% (Grupo I), conforme se verifica na Tabela 3.

Tabela 3 Composição dos três grupos formados (sexo)

\begin{tabular}{|llll|}
\hline Gênero & Grupo 1 & Grupo 2 & Grupo 3 \\
\hline Masculino & $77(72,6 \%)$ & $47(54,7 \%)$ & $49(57 \%)$ \\
\hline Feminino & $29(27,4 \%)$ & $39(45,3 \%)$ & $37(43 \%)$ \\
\hline Total & $\mathbf{1 0 6 ( 1 0 0 \% )}$ & $\mathbf{8 6}(\mathbf{1 0 0 \% )}$ & $\mathbf{8 6 ( 1 0 0 \% )}$ \\
\hline
\end{tabular}

Aplicando-se o teste qui-quadrado em relação ao sexo obteve-se um p-valor $=$ o,or8, indicando haver diferença, estatisticamente significante, na composição dos grupos. 
Em relação à idade dos respondentes, verificou-se uma grande proximidade dos valores, tanto das médias, como dos desvios-padrão dos indivíduos dos três grupos, conforme presente na Tabela 4. Aplicando-se o teste de KruskalWallis, verificou-se a não existência de diferença significativa de idade entre os grupos tendo em vista que se obteve $\mathrm{p}=0,834$.

Tabela 4 Composição dos três grupos formados (idade)

\begin{tabular}{|llll|}
\hline Idade & Grupo 1 & Grupo 2 & Grupo 3 \\
\hline Média & 20,92 & 21,22 & 21,1 \\
\hline Desvio-padrão & 2,82 & 2,79 & 2,63 \\
\hline
\end{tabular}

Em relação à variável período do curso, verificou-se um número superior de alunos do período noturno nos três grupos, desde 60,5\% (Grupo 2) até $67,9 \%$ (Grupo 3), conforme apresentado na Tabela 5.

Tabela 5 Composição dos três grupos formados (período do curso)

\begin{tabular}{|llll|}
\hline Período do curso & Grupo 1 & Grupo 2 & Grupo 3 \\
\hline Diurno & $34(32,1 \%)$ & $34(39,5 \%)$ & $28(32,6 \%)$ \\
\hline Noturno & $72(67,9 \%)$ & $52(60,5 \%)$ & $58(67,4 \%)$ \\
\hline Total & $\mathbf{1 0 6 ( 1 0 0 \% )}$ & $\mathbf{8 6}(\mathbf{1 0 0 \% )}$ & $\mathbf{8 6}(\mathbf{1 0 0 \% )}$ \\
\hline
\end{tabular}

Aplicando-se o teste qui-quadrado, em relação ao período do curso, obtevese um p-valor $=0,501$, indicando não haver diferença, estatisticamente significante, na composição dos grupos.

Pelo fato de terem sido utilizadas somente disciplinas pertencentes ao primeiro e ao segundo ano do curso, verificou-se, nos três grupos formados, uma ampla participação de alunos que apenas cursam a faculdade, conforme consta na Tabela 6. 
Tabela 6 Composição dos três grupos formados (atividade desempenhada)

\begin{tabular}{|llll|}
\hline $\begin{array}{l}\text { Atividade } \\
\text { desempenhada }\end{array}$ & Grupo 1 & Grupo 2 & Grupo 3 \\
\hline Estuda somente & $49(46,2 \%)$ & $39(45,3 \%)$ & $39(45,3 \%)$ \\
\hline Estágio e estuda & $37(34,9 \%)$ & $22(25,6 \%)$ & $29(33,7 \%)$ \\
\hline $\begin{array}{l}\text { Iniciação científica e } \\
\text { estuda }\end{array}$ & $0(0 \%)$ & $6(7,0 \%)$ & $7(8,1 \%)$ \\
\hline $\begin{array}{l}\text { Trabalha meio } \\
\text { período e estuda }\end{array}$ & $6(5,7 \%)$ & $3(3,5 \%)$ & $3(3,5 \%)$ \\
\hline $\begin{array}{l}\text { Trabalha período } \\
\text { integral e estuda }\end{array}$ & $13(12,3 \%)$ & $15(17,4 \%)$ & $8(9,3 \%)$ \\
\hline Outra & $1(0,9 \%)$ & $1(1,2 \%)$ & $0(0 \%)$ \\
\hline Total & $106(100 \%)$ & $86(100 \%)$ & $86(100 \%)$ \\
\hline
\end{tabular}

Aplicando-se o teste qui-quadrado em relação à atividade desempenhada obteve-se um $p$-valor $=0,196$, indicando não haver diferença, estatisticamente significante, na composição dos grupos.

Em relação à distribuição da área de maior interesse do aluno, verificouse que a preferência pela área de Marketing foi a superior tanto no Grupo I quanto no Grupo 2; por sua vez, o Grupo 3 apresentou um valor superior em relação à área de Finanças (47,7\%) e o menor valor em relação à área de Recursos Humanos (7\%), em comparação com os demais grupos, conforme se verifica na Tabela 7 .

Tabela 7 Composição dos três grupos formados (área de interesse)

\begin{tabular}{|llll|}
\hline Área de interesse & Grupo 1 & Grupo 2 & Grupo 3 \\
\hline Finanças & $35(33 \%)$ & $16(18,6 \%)$ & $41(47,7 \%)$ \\
\hline Marketing & $46(43,4 \%)$ & $36(41,9 \%)$ & $26(30,2 \%)$ \\
\hline Produção & $5(4,7 \%)$ & $9(10,5 \%)$ & $6(7 \%)$ \\
\hline Recursos humanos & $15(14,2 \%)$ & $17(19,8 \%)$ & $6(7 \%)$ \\
\hline Outra & $5(4,7 \%)$ & $8(9,3 \%)$ & $7(8,1 \%)$ \\
\hline Total & $106(100 \%)$ & $86(100 \%)$ & $86(100 \%)$ \\
\hline
\end{tabular}


Aplicando-se o teste qui-quadrado em relação à área de interesse, obteve-se um $p$-valor $=0,004$, que indica haver diferença, estatisticamente significante, na composição dos grupos.

Dessa maneira, de modo mais contundente, apenas a variável área de interesse mostrou-se como possível variável diferenciadora dos grupos formados, dadas as diferenciações entre o número de alunos que declaram interesse por uma determinada área. Antes de se iniciar a análise dos resultados referentes tanto às dimensões atitudinais, quanto em relação às dimensões motivacionais nos clusters formados, faz-se importante apresentar os valores para o $\alpha$ de Cronbach, uma vez que servem como parâmetros para análise da confiabilidade.

Tabela 8 Valores do Alpha de Cronbach para a sATs, encontrados na pesquisa

\begin{tabular}{|lll|}
\hline Dimensões da atitude & $N^{\circ}$ de itens & valores do $\alpha$ de Cronbach \\
\hline 1. Afeto & 6 & 0,833 \\
\hline 2. Competência Cognitiva & 6 & 0,705 \\
\hline 3. Valor & 9 & 0,823 \\
\hline 4. Dificuldade & 7 & 0,632 \\
\hline
\end{tabular}

É possível concluir que as dimensões da escala sats apresentaram confiabilidade considerável, sendo importante relatar que apenas a dimensão Dificuldade apresentou um valor para o $\alpha$ de Cronbach abaixo de o,7, sendo justificável tal valor por tratar-se de uma dimensão psicológica. Resultados semelhantes encontram-se em Mantovani e Viana (2007), com um $\alpha=0,59$, em Wiberg (2009), com um $\alpha=0,65$ e em Chiesi e Primi (2010), com um $\alpha$ pré-teste $=0,6 \mathrm{I}$ e um $\alpha$ pós-teste $=0,60$, tendo em vista apresentarem valores de $\alpha$ de Cronbach inferiores a $0,7 .{ }^{\star \star \star}$ 
Tabela 9 Valores do Alpha de Cronbach para a EmA, encontrados na pesquisa

\begin{tabular}{|lll|}
\hline Dimensões do continuum da motivação & No de itens & valores do $\alpha$ de Cronbach \\
\hline 1. Motivação intrínseca para saber & 4 & 0,830 \\
\hline 2. Motivação intrínseca para realização & 4 & 0,779 \\
\hline 3. Motivação intrínseca para vivenciar estímulos & 4 & 0,757 \\
\hline 4. Motivação extrínseca - identificação & 4 & 0,667 \\
\hline 5. Motivação extrínseca - introjeção & 4 & 0,785 \\
\hline 6. Motivação extrínseca - controle externo & 4 & 0,757 \\
\hline 7. Desmotivação & 4 & 0,769 \\
\hline
\end{tabular}

Conclui-se, com base na exposição dos resultados encontrados, que as dimensões da escala EMA apresentaram confiabilidade considerável, sendo importante relatar que apenas a dimensão Motivação Extrínseca - identificação apresentou um valor para o $\alpha_{c}$ de Cronbach abaixo de o,7, sendo justificável tal valor por tratar-se de dimensão psicológica. Outra questão, que deve ser levada em consideração, refere-se ao tamanho das amostras utilizadas, tendo em vista que influenciam no valor apurado de $\alpha_{\mathrm{c}}$.

No que tange à aplicação da análise de cluster, é importante analisar a formação dos grupos em relação às dimensões atitudinais perante a Estatística. No que concerne ao valor obtido em cada dimensão atitudinal, salienta-se que este foi obtido por meio do cálculo da média dos valores assinalados no questionário em relação à cada dimensão. Desse modo, o mínimo valor possível seria $\mathrm{r}$ e o máximo seria 7 . Em relação à média das dimensões, foi possível observar que o Grupo 3 apresentou maiores valores para todas as dimensões, enquanto o Grupo 2 apresentou os menores valores, também para todas as dimensões, conforme apresentado na Tabela Io. 
ATITUDE E MOTIVAÇÃO EM RELAÇÃO AO DESEMPENHO ACADÊMICO DE ALUNOS DO CURSO DE GRADUAÇÃO EM ADMINISTRAÇÃO EM DISCIPLINAS DE ESTATÍSTICA: FORMAÇÃO DE CLUSTERS

Tabela 10 Dimensões atitudinais nos três grupos formados

\begin{tabular}{|llll|}
\hline Dimensões atitudinais & Grupo 1 & Grupo 2 & Grupo 3 \\
\hline Média (desvio-padrão) & & & \\
\hline Afeto & $4,43(1,21)$ & $3,02(1,01)$ & $5,09(0,85)$ \\
\hline Competência cognitiva & $5,08(0,92)$ & $3,98(0,89)$ & $5,39(0,66)$ \\
\hline Valor & $5,26(0,92)$ & $5,03(1,15)$ & $5,88(0,65)$ \\
\hline Dificuldade & $3,60(0,81)$ & $2,67(0,69)$ & $3,38(0,72)$ \\
\hline
\end{tabular}

Foi realizada, em um segundo momento, a análise sobre possíveis diferenças entre os grupos no que concerne às dimensões atitudinais. Para tanto, foi utilizado o teste não paramétrico de Kruskal-Wallis, com as seguintes hipóteses:

Ho: Os grupos apresentam médias iguais em relação às dimensões atitudinais;

Hı: Pelo menos um grupo apresenta média diferente dos demais em relação às dimensões atitudinais.

Os resultados encontram-se na Tabela II.

Tabela 11 Resultado do teste não paramétrico de Kruskal-Wallis para as dimensões atitudinais

\begin{tabular}{|lll|}
\hline Dimensões atitudinais & $\mathrm{p}$-valor & Decisão \\
\hline Afeto & 0 & rejeita-se \\
\hline Competência cognitiva & 0 & rejeita-se \\
\hline Valor & 0 & rejeita-se \\
\hline Dificuldade & 0 & rejeita-se \\
\hline
\end{tabular}

Considerando os valores obtidos (p-valores), é possível afirmar que, pelo menos um grupo difere dos demais em relação a todas as dimensões atitudinais, servindo, portanto, como uma considerável variável de formação dos grupos.

Quando se combinaram os grupos para uma análise aos pares, observouse que, em relação à dimensão Afeto, os três grupos diferem entre si. Já em relação à dimensão Competência cognitiva observou-se que os grupos I 
e 2 e os grupos 2 e 3 são diferentes entre si. No que concerne à dimensão Valor, observou-se que os grupos i e 3 e os grupos 2 e 3 diferem entre si. E, por último, em relação à dimensão Dificuldade, observou-se que os grupos I e 2 e os grupos 2 e 3 diferem entre si.

É interessante destacar que os grupos 2 e 3 se diferenciaram em relação a todas as dimensões atitudinais perante a Estatística.

Analisou-se também a formação dos grupos em relação às dimensões motivacionais. No que concerne ao valor obtido em cada dimensão motivacional, salienta-se que este foi obtido por meio do cálculo da média dos valores assinalados no questionário em relação a cada dimensão. Desse modo, o mínimo valor possível seria i e o máximo seria 7. Conforme apresentado na Tabela ı2, contemplando a análise descritiva das dimensões motivacionais, considerando a média e o desvio-padrão.

Tabela 12 Análise descritiva das dimensões motivacionais nos grupos formados

\begin{tabular}{|llll|}
\hline Dimensões motivacionais & Grupo 1 & Grupo 2 & Grupo 3 \\
\hline Média (desv. padrão) & & & \\
\hline 1. Motivação intrínseca para saber & $4,66(1,04)$ & $5,63(0,76)$ & $5,79(0,72)$ \\
\hline 2. Motivação intrínseca para realização & $3,45(0,95)$ & $4,96(0,91)$ & $5,22(0,79)$ \\
\hline 3. Motivação intrínseca para vivenciar estímulos & $3,67(1,09)$ & $4,81(0,97)$ & $4,88(0,85)$ \\
\hline 4. Motivação extrínseca - identificação & $5,27(0,92)$ & $6,14(0,71)$ & $6,16(0,60)$ \\
\hline 5. Motivação extrínseca - introjeção & $3,08(1,13)$ & $4,59(1,43)$ & $4,43(1,00)$ \\
\hline 6. Motivação extrínseca - controle externo & $5,35(1,17)$ & $6,20(0,82)$ & $5,91(0,71)$ \\
\hline 7. Desmotivação & $2,52(1,17)$ & $1,66(0,81)$ & $1,61(0,76)$ \\
\hline
\end{tabular}

Para a melhor análise das possíveis diferenciações existentes em relação às dimensões atitudinais entre os grupos, fez-se a aplicação do teste não paramétrico de Kruskal-Wallis, com as seguintes hipóteses:

Ho: Os grupos apresentam médias iguais em relação às dimensões motivacionais;

Hı: Pelo menos um grupo apresenta média diferente dos demais em relação às dimensões motivacionais. 
ATITUDE E MOTIVAÇÃO EM RELAÇÃO AO DESEMPENHO ACADÊMICO DE ALUNOS DO CURSO DE GRADUAÇÃO EM ADMINISTRAÇÃO EM DISCIPLINAS DE ESTATÍSTICA: FORMAÇÃO DE CLUSTERS

A Tabela I3 apresenta os resultados do teste Kruskal-Wallis.

Tabela 13 Análise descritiva das dimensões motivacionais nos grupos formados

\begin{tabular}{|lll|}
\hline Dimensões motivacionais & p-valor & Decisão \\
\hline 1. Motivação intrínseca para saber & 0 & rejeita-se \\
\hline 2. Motivação intrínseca para realização & 0 & rejeita-se \\
\hline 3. Motivação intrínseca para vivenciar estímulos & 0 & rejeita-se \\
\hline 4. Motivação extrínseca - identificação & 0 & rejeita-se \\
\hline 5. Motivação extrínseca - introjeção & 0 & rejeita-se \\
\hline 6. Motivação extrínseca - controle externo & 0 & rejeita-se \\
\hline 7. Desmotivação & 0 & rejeita-se \\
\hline
\end{tabular}

Quando se combinaram os grupos para uma análise aos pares, observouse que os grupos I e 2 apresentaram diferenciação em relação a todas as dimensões motivacionais; em relação ao Grupo i e 3, também se verificou diferenciação em relação a todas as dimensões motivacionais; e, no que concerne à comparação entre os grupos 2 e 3, apenas em relação à dimensão Motivação extrínseca - controle externo não se verificou diferenciação, sendo que em todas as outras dimensões vislumbrou-se diferenciação.

Procedeu-se à análise referente ao desempenho dos alunos pertencentes aos grupos formados, conforme apresentado na Tabela I4. O Grupo 3 apresentou a maior média na nota da disciplina $(8,49)$, bem como maior média em relação à autopercepção de desempenho $(7,42)$, sendo importante considerar ainda que apresentou os menores valores para o desviopadrão, tanto em relação à nota na disciplina, quanto à autopercepção do desempenho.

Tabela 14 Análise descritiva do desempenho dos grupos

\begin{tabular}{|llll|}
\hline Desempenho (média/desvio-padrão) & Grupo 1 & Grupo 2 & Grupo 3 \\
\hline Nota na disciplina & $7,09(2,00)$ & $7,13(1,93)$ & $8,49(1,34)$ \\
\hline Autopercepção & $6,11(1,82)$ & $4,77(1,66)$ & $7,42(1,03)$
\end{tabular}


Aplicando-se o teste de Kruskal-Wallis, verificou-se a existência de diferença significativa tanto em relação à nota como em relação à autopercepção de desempenho entre os grupos, tendo em vista que para ambos os teste obteve-se $\mathrm{p}<0,000$.

Observando-se o Gráfico I, verifica-se como o Grupo 3 apresentou um resultado superior, bem como uma menor dispersão, em relação à nota obtida na disciplina.

Gráfico 1 Box-plot das notas dos três grupos formados

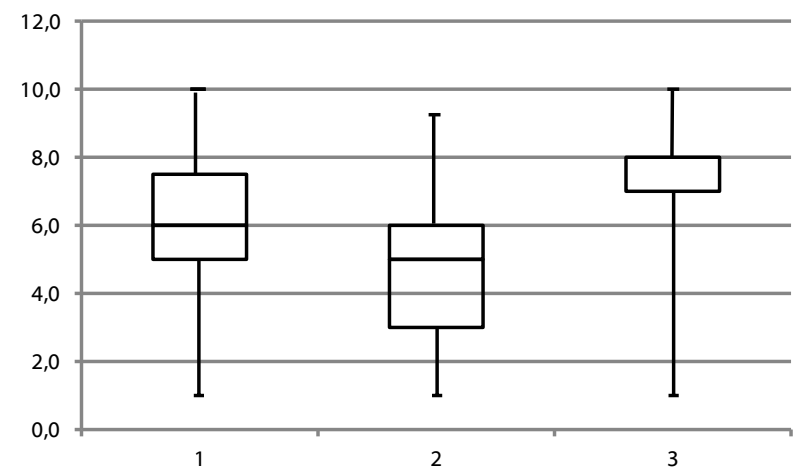

Observando-se o Gráfico 2, verifica-se como o Grupo 3 apresentou também um resultado superior, bem como uma menor dispersão, em relação à autopercepção de desempenho.

Gráfico 2 Box-plot da autopercepção dos três grupos formados

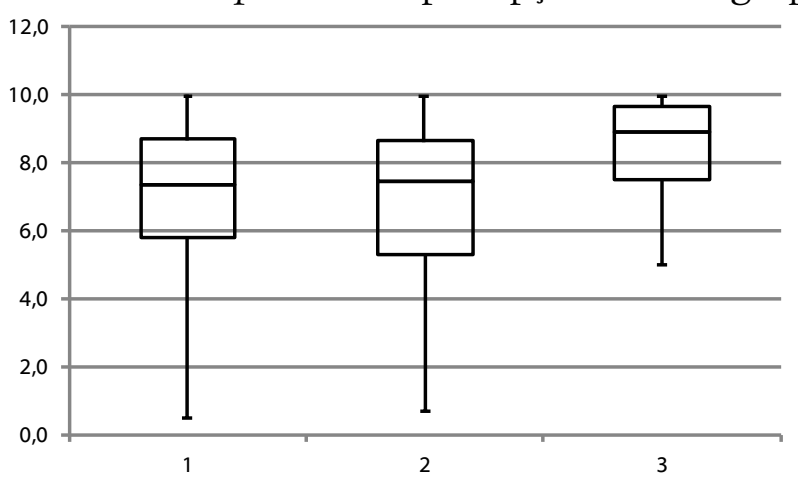


Portanto, dada a inclusão das variáveis: atitudes perante a Estatística (Afeto, Competência cognitiva, Valor e Dificuldade), motivacionais acadêmicas (Motivação intrínseca para saber, Motivação intrínseca para realização, Motivação intrínseca para vivenciar estímulos, Motivação extrínseca - identificação, Motivação extrínseca - introjeção, Motivação extrínseca - controle externo e Desmotivação), nota na disciplina e autopercepção de desempenho, foi possível estabelecer a formação de 3 grupos com as seguintes características gerais, conforme Quadro I.

Quadro 1 Resumo das características dos grupos

\begin{tabular}{|c|c|c|c|}
\hline & Grupo 1 & Grupo 2 & Grupo 3 \\
\hline \multicolumn{4}{|l|}{ Caracterização geral } \\
\hline Gênero & $\begin{array}{l}\text { maior predominância } \\
\text { masculina }\end{array}$ & & \\
\hline Área de interesse & & & $\begin{array}{l}\text { predominância da } \\
\text { área financeira }\end{array}$ \\
\hline \multicolumn{4}{|c|}{ Atitude perante a Estatística } \\
\hline Afeto & & $\begin{array}{l}\text { menor média } \\
\text { observada }\end{array}$ & $\begin{array}{l}\text { maior média } \\
\text { observada }\end{array}$ \\
\hline $\begin{array}{l}\text { Competência } \\
\text { Cognitiva }\end{array}$ & & $\begin{array}{l}\text { menor média } \\
\text { observada }\end{array}$ & $\begin{array}{l}\text { maior média } \\
\text { observada }\end{array}$ \\
\hline Valor & & $\begin{array}{l}\text { menor média } \\
\text { observada }\end{array}$ & $\begin{array}{l}\text { maior média } \\
\text { observada }\end{array}$ \\
\hline Dificuldade & & $\begin{array}{l}\text { menor média } \\
\text { observada }\end{array}$ & $\begin{array}{l}\text { maior média } \\
\text { observada }\end{array}$ \\
\hline \multicolumn{4}{|l|}{ Motivação acadêmica } \\
\hline $\begin{array}{l}\text { Motivação intrínseca } \\
\text { para saber }\end{array}$ & $\begin{array}{l}\text { menor média } \\
\text { observada }\end{array}$ & & $\begin{array}{l}\text { maior média } \\
\text { observada }\end{array}$ \\
\hline $\begin{array}{l}\text { Motivação intrínseca } \\
\text { para realização }\end{array}$ & $\begin{array}{l}\text { menor média } \\
\text { observada }\end{array}$ & & $\begin{array}{l}\text { maior média } \\
\text { observada }\end{array}$ \\
\hline $\begin{array}{l}\text { Motivação intrínseca } \\
\text { para vivenciar } \\
\text { estímulos }\end{array}$ & $\begin{array}{l}\text { menor média } \\
\text { observada }\end{array}$ & & $\begin{array}{l}\text { maior média } \\
\text { observada }\end{array}$ \\
\hline $\begin{array}{l}\text { Motivação extrínseca - } \\
\text { identificação }\end{array}$ & $\begin{array}{l}\text { menor média } \\
\text { observada }\end{array}$ & & $\begin{array}{l}\text { maior média } \\
\text { observada }\end{array}$ \\
\hline $\begin{array}{l}\text { Motivação extrínseca - } \\
\text { introjeção }\end{array}$ & $\begin{array}{l}\text { menor média } \\
\text { observada }\end{array}$ & & $\begin{array}{l}\text { maior média } \\
\text { observada }\end{array}$ \\
\hline $\begin{array}{l}\text { Motivação extrínseca - } \\
\text { controle externo }\end{array}$ & $\begin{array}{l}\text { menor média } \\
\text { observada }\end{array}$ & $\begin{array}{l}\text { maior média } \\
\text { observada }\end{array}$ & \\
\hline
\end{tabular}




\begin{tabular}{|llll|}
\hline & Grupo 1 & Grupo 2 & Grupo 3 \\
\hline Desmotivação & $\begin{array}{l}\text { maior média } \\
\text { observada }\end{array}$ & $\begin{array}{l}\text { menor média } \\
\text { observada }\end{array}$ \\
\hline Desempenho & menor média & \\
\hline observada & & maior média \\
\hline Autopercepção & observada \\
\hline
\end{tabular}

Conclui-se, assim, que o Grupo 3, grupo com maior concentração de alunos com interesse na área de Finanças, apresentou a maior média em relação à nota e, à autopercepção de desempenho, bem como em relação a todas as dimensões atitudinais perante a Estatística e as dimensões da motivação acadêmica, exceto no que concerne à Motivação Extrínseca - controle externo e Desmotivação.

Observa-se que em relação à preferência pela área de marketing, os grupos i e 2 apresentaram valores superiores ao Grupo 3, no entanto, não foi possível a realização de uma associação entre tal preferência e o desempenho dos alunos.

Em relação à associação da atitude perante a Estatística e o desempenho acadêmico observa-se que os resultados foram ao encontro dos vislumbrados por Cashin e Elmore (2005) e por Pimentel (2009). Em relação à associação da motivação acadêmica quanto ao desempenho acadêmico observa-se que os resultados obtidos foram similares aos descritos por Smith, Davy e Rosenber (2010).

Por último, com base na formação dos três grupos, foi possível estabelecer, de acordo com o Quadro 2, o seguinte perfil para cada um. 
Quadro 2 Perfil dos grupos formados

\begin{tabular}{|c|c|c|}
\hline Grupo 1 & Grupo 2 & Grupo 3 \\
\hline $\begin{array}{l}\text { Grupo com uma postura inter- } \\
\text { mediária em relação à atitude } \\
\text { perante a Estatística; com baixa } \\
\text { motivação, tanto intrínseca } \\
\text { como extrínseca, aliado a uma } \\
\text { maior desmotivação, apre- } \\
\text { sentou a menor média (nota) } \\
\text { observada entre os três grupos } \\
\text { formados. }\end{array}$ & $\begin{array}{l}\text { Grupo com uma baixa atitude } \\
\text { perante a Estatística, com des- } \\
\text { taque para a motivação extrín- } \\
\text { seca por controle externo; } \\
\text { apresentou a menor pontua- } \\
\text { ção em relação à autopercep- } \\
\text { ção de desempenho. }\end{array}$ & $\begin{array}{l}\text { Grupo com uma postura muito } \\
\text { positiva em relação à atitude } \\
\text { perante a Estatística, bem } \\
\text { como motivado academica- } \\
\text { mente; apresentou a maior } \\
\text { média, tanto em relação à nota, } \\
\text { como em relação à autoper- } \\
\text { cepção de desempenho. }\end{array}$ \\
\hline
\end{tabular}

Considerando o exposto anteriormente, é possível observar, portanto, os modos particulares de interação entre motivação, atitude e desempenho nos grupos formados. 


\section{CONSIDERAÇÕES FINAIS}

Na busca pela formação de grupos homogêneos em relação à motivação acadêmica, atitude perante a Estatística e desempenho, verificou-se a formação de 3 grupos. No que concerne às variáveis de caracterização, apenas a área de interesse mostrou-se como possível variável diferenciadora, dada a composição dos 3 grupos.

No que concerne à atitude perante a Estatística, os três grupos apresentaram diferentes valores para a dimensão Afeto. Em relação à dimensão Competência cognitiva e Dificuldade, verificou-se que os grupos I / 2 e 2 / 3 diferiram entre si. Em relação à dimensão Valor, verificou-se que os grupos I / 3 e 2 / 3 diferiram entre si. É notório, portanto, que os grupos 2 e 3 diferiram em relação a todas as dimensões.

Em relação à motivação acadêmica, verificou-se que todos os grupos apresentaram diferenças em relação às dimensões motivacionais, exceto os grupos 2 e 3 em relação à Motivação extrínseca - controle externo.

Quando se analisou a formação dos grupos em relação ao desempenho, verificou-se que o Grupo 3 apresentou valores superiores e menos dispersos, tanto no que concerne à nota, quanto em relação à autopercepção de desempenho. Neste sentido, dada a análise dos resultados, foi possível concluir que, de modo geral, o grupo de alunos com maior interesse na área de Finanças, com as maiores pontuações tanto em relação à atitude perante a Estatística como em relação à motivação acadêmica (exceto Desmotivação), apresentaram os maiores valores para o desempenho (nota e autopercepção).

Dessa maneira, conclui-se que os alunos que apresentaram grande interesse em finanças mostraram-se mais motivados e com atitudes mais positivas em relação à Estatística do que os outros grupos de interesse. Neste sentido, surge como proposta uma maior integração no ensino das duas áreas, realização esta que beneficiaria tanto o ensino de Estatística, bem como o ensino de Finanças.

Tais resultados contribuem para a academia no sentido de oferecerem um novo olhar sobre o ensino de Estatística em relação ao curso de 
Administração, tendo em vista ratificar os resultados que associam uma maior atitude, bem como uma maior motivação intrínseca em relação ao desempenho; porém, insere como grande elemento de formação de tal grupo os alunos que apresentam um maior interesse na área de finanças.

Como contribuição para a prática, verifica-se a necessidade de maior exploração da intersecção existente entre Estatística e finanças, potencializando, desse modo, a associação vislumbrada no presente artigo entre as duas áreas.

Em relação às limitações do trabalho, a presente pesquisa, objetivando explicar o desempenho do aluno de graduação em Administração nas disciplinas de Estatística, por meio de um modelo matemático, reduziu a questão a poucas variáveis, não considerando, portanto, aspectos como a experiência prévia dos alunos, e instrumentos de ensino utilizados pelo docente da disciplina, entre outros.

Outra limitação em relação ao trabalho refere-se ao instrumento por meio do qual os dados foram coletados, tendo em vista que os alunos não puderam contribuir com questões não vislumbradas pelas escalas. Desse modo, a pesquisa ficou adstrita às questões presentes nos referidos instrumentos.

Destaca-se também a existência de limitações concernentes ao escopo do trabalho, como, por exemplo, o fato de serem investigadas somente universidades públicas, além de a pesquisa ter se utilizado de uma amostra de apenas 278 estudantes, bem como professores distintos poderem ter-se utilizado formas muito diferentes de ensino e de avaliação, fator este que, consequentemente, pode afetar a postura do aluno perante a Estatística.

Considerando os resultados obtidos na presente pesquisa, abre-se um grande espectro de possibilidades para o desenvolvimento de novas pesquisas.

Sugere-se também a aplicação do mesmo método em outros cursos de graduação, tanto na área de negócios (Economia, contabilidade, atuária), como em outras áreas, buscando encontrar relações como a área de Finanças e a área de Estatística.

Podem ser realizadas novas abordagens metodológicas, considerando 
as mesmas variáveis, como, por exemplo, a utilização de uma pesquisa qualitativa, ou, no campo da pesquisa quantitativa, com o uso de modelagem de equações estruturais.

Outra possibilidade de pesquisa refere-se ao estudo das metodologias de ensino de Estatística empregadas e o modo como elas podem interferir no desempenho do aluno. Por último, sugere-se ainda a possibilidade de desenvolvimento de um estudo de caso para análise dos fatores influenciadores do desempenho, podendo ser aplicado tanto em uma instituição (estudo de caso único), como também em várias instituições (estudo multicaso), permitindo, assim, analisar com maior profundidade a referida problemática. 


\section{REFERÊNCIAS}

ARA, A. B. O ensino de Estatística e a busca do equilíbrio entre os aspectos determinísticos e aleatórios da realidade. 2006. 86 f. Tese (Doutorado em Educação) - Faculdade de Educação, Universidade de São Paulo, São Paulo, 2006.

BARKOUKIS, V.; TSORBATZOUDIS, H.; GROUIOS, G.; SIDERIDIS, G. The assessment of intrinsic and extrinsic motivation and amotivation: Validity and reliability of the Greek version of the Academic Motivation Scale. Assessment in Education: Principles, Policy \& Practice, v. 15, n. 1, p. 39-55, 2008.

BASTOS, R.; OLIVEIRA, F. M.; OLIVEIRA, J. P. M. Modelagem do processo de tomada de decisão para alocação de recursos. Revista de Administração, v. 33, n. 3, p. 73-82, 1998.

BROUSE, C.; BASCH, C,; LEBLANC, M.; MCKNIGHT, K.; LEI, T. College students' academic motivation: Differences by gender, class, and source of payment. College Quarterly, Seneca, v. 13, n. 1, 2010. Disponível em: <http://www.senecac.on.ca/ quarterly/2010-vol13-num01-winter/brouse-basch-leblanc-mcknight-lei.html>. Acesso em: 29/06/2011.

BROWN, E.; KASS, R. What Is Statistics? The American Statistician, Alexandria, v. 63, p. 105-123, 2009. Disponível em: <http://www.scielo.org.za/scielo. php?pid=S20710763201 0000100009\&script=sci_arttext $>$. Acesso em: 25/01/2011.

BURAND, C. Good Statistics are key to management. American Agent \& Broker, v. 81, n. 7, p. 14-16, 2009.

CARLSON, K. A.; WINQUIST, J. R. Evaluating an active learning approach to teaching introductory statistics: A classroom workbook approach. Journal of Statistics Education, Alexandria, v. 19, n.1, 2011. Disponível em: <www.amstat.org/ publications/jse/v19n1/carlson.pdf > . Acesso em: 10/06/2011.

CASHIN, S.E.; ELMORE, P.B. The Survey of Attitudes Toward Statistics Scale: A construct validity study. Educational and Psychological Measurement, v. 65, p. 509-524, 2005.

CAVENAGHI, A. R. Uma perspectiva autodeterminada da motivação para aprender língua estrangeira no contexto escolar. Ciências \& Cognição, Rio de Janeiro, v. 14, n. 2, 2009. Disponível em: < http://pepsic.bvsalud.org/scielo.php?pid=S180658212009000200017\&script=sci_arttext $>$. Acesso em: 20/06/2011.

CHIESI, F.; PRIMI, C. Assessing statistics attitudes among college students: Psychometric properties of the Italian version of the Survey of Attitudes toward Statistics (SATS). Learning and Individual Differences, v. 19, n.1, p. 309-313, 2010.

COX, E. B. The Teaching of Statistics in Business Schools - A Summary Report. The American Statistician, v. 19, n. 1, p. 17-19, 1965. 
DARGAHY-NOUBARY, G. R.; GROWNEY, H. A. S. Risk-A Motivating Theme for an Introductory Statistics Course. Structural Equation Modeling, v. 52, n. 1, p. 44-48, 1998.

DAVIS, G. P. The Relationship Between Racial Identity, Motivation, and the Academic Performance of African American Students at a Predominately White Institution. 2009. $184 \mathrm{f}$. Tese (Doutorado em Educação) - George Washington University, Washington, 2009. DECI, E.; RYAN, R. Intrinsic Motivation and Self-Determination in Human Behavior. Plenum Press: New York, 1985.

DECI, E.; RYAN, R. The "What" and "Why” of Goal Pursuits: Human Needs and the Self-Determination of Behavior. Psychological Inquiry, v. 11, n. 4, p. 227-268, 2000. Disponível em: <http://www.psych.rochester.edu/SDT/documents/2000_ DeciRyan_PIWhatWhy.pdf $>$. Acesso em: 22/01/2011.

DEVANEY, T. A. Anxiety and Attitude of Graduate Students in On-Campus vs.Online Statistics Courses. Journal of Statistics Education, v. 18, n.1, 2010. Disponível em: < www.amstat.org/publications/jse/v18n1/devaney.pdf > . Acesso em: 21/01/2011.

GAL, I.; GINSBURG, L.; SCHAU, C. Monitoring attitudes and beliefs in Statistics Education. In: I. Gal \& J. B. Garfield (Orgs.) The assessment challenge in Statistics Education. Washington: IOS, p.37-51, 1997. Disponível em: < http://www.stat. auckland.ac.nz/ iase/publications/assessbkref.>. Acesso em: 17/07/2011.

GOULD, R. Variability: one statistician's view. Statistic education research journal, v. 3, n.2, p. 7-16, 2004. Disponível em: <http://www.stat.auckland.ac.nz/ iase/serj/ SERJ3(2)_Gould.pdf $>$. Acesso em: 20/01/2012.

GUIMARÃES, S.; BUROCHOVITCH, E. O Estilo Motivacional do Professor e a Motivação Intrínseca dos Estudantes: Uma Perspectiva da Teoria da Autodeterminação. Psicologia: Reflexão e Crítica, v.17, n. 2, p.143-150, 2004.

HAIR, J. F.; BABIN, B.; MONEY, A. H.; SAMOUEL, P. Fundamentos de métodos de pesquisa em administração. 1.ed. Porto Alegre: Bookman, 2005. $471 \mathrm{p}$.

HEGARTY, N. The Application of the Academic Motivation Scale to Graduate School Students. The Journal of Human Resource and Adult Learning, v. 6, n. 2, p. 48-55, 2010.

LAVENDER, M. M. A comparison of academic motivation of academically prepared and academically unprepared community college students. 2005. $88 \mathrm{f}$. Tese (Doutorado em Educação) - College of Education, Florida State University, Florida, 2005.

MANTOVANI, D. M. N. Método para a implementação e o acompanhamento de atividades a distância em disciplinas de Estatística: um estudo de caso. 2008. $247 \mathrm{f}$. Dissertação (Mestrado em Administração de Organizações) - Faculdade de Economia, Administração e Contabilidade de Ribeirão Preto, Universidade de São Paulo, Ribeirão Preto, 2008. 
ATITUDE E MOTIVAÇÃO EM RELAÇÃO AO DESEMPENHO ACADÊMICO DE ALUNOS DO CURSO DE GRADUAÇÃO EM ADMINISTRAÇÃO EM DISCIPLINAS DE ESTATÍSTICA: FORMAÇÃO DE CLUSTERS

MANTOVANI, D. M. N.; VIANA, A. B. N. Ensino de estatística para cursos de graduação em administração: novas perspectivas. In: SEMINÁRIOS EM ADMINISTRAÇÃO, 7., 2004, Brasília. Anais... São Paulo: Seminários em Administração FEA-USP, 2007.

MAXIMIANO, A. C. A. Teoria geral da Administração: da revolução urbana à revolução digital. 4 ed. São Paulo: Atlas, 2004.

MCALEVEY, L.; SULLIVAN, C. Making statistics more efective for business? International Journal of Mathematical Education in Science and Technology, v. 32, n. 3, p. 425-438, 2001.

MENG, X. Desired and feared - what do we do now and over the next 50 years? The American Statistical Association, v. 63, n. 3, p. 202-210, 2009.

MILAGRE, R. A. Estatística: uma proposta de ensino para os cursos de Administração de Empresas. 2001. 160 f. Dissertação (Mestrado em Engenharia de Produção) - Engenharia de Produção, Universidade Federal de Santa Catarina, Florianópolis, 2001.

NOLAN, D.; SPEED, T. P.; Teaching Statistics Theory Throught Applications. The American Statistical,v. 53, n.4, p. 370-375, 1999.

OLIVEIRA, S. L. Tratado de metodologia científica: projetos de pesquisas, TGI, TCC, monografias, dissertações e teses. 2.ed. São Paulo: Pioneira, 1999.

PAIVA, W. P. Avaliação de habilidades para a tomada de decisão em administração de empresas: um estudo descritivo com alunos de graduação da FEA-USP. 2002. 115 f. Dissertação (Mestrado em Administração) - Faculdade de Economia, Administração e Contabilidade, Universidade de São Paulo, São Paulo, 2002.

PIMENTEL, A. C. S. Análise da influência do estilo de aprendizagem e da atitude em disciplinas de estatística da FEA-RP. 2009. 187f. Dissertação (Mestrado em Administração de Organizações) - Faculdade de Economia, Administração e Contabilidade de Ribeirão Preto, Universidade de São Paulo, Ribeirão Preto, 2009.

REEVE, J.; DECI, E.; RYAN, R. Self-Determination Theory: A dialectical framework for understand sociocultural influences on student motivation. 3.ed. Connecticut: Information Age Publishing, 2004. 368 p. Editado por Dennis McInerney e Shawn Van Ette.

RICHARDSON, R. J. Pesquisa social: métodos e técnicas. 3. ed. São Paulo: Atlas, I999. $334 \mathrm{p}$.

SMITH, K.; DAVY, J.; ROSENBER, G. An examination of the validity of the academic motivation scale with a United States business student sample. Psychological Reports. Missoula, v. I06, n. 2, p. 323-34I, 2010. 
SCHAU, C.; STEVENS, J.; DAULPHINEE, T. L.; VECCHIO, A. D. The development and validation of the survey of attitudes toward statistics. Educational and psychological measurement, v. 55, n.5, p. 868-875, 1995.

SIMON, H. A. The new science of management decision. New York: Harper \& Brothers Publishers, 1960.

SINGH, K.; GRANVILLE, M.; DIKA, S. Mathematics and Science Achievement: Effects of Motivation, Interest, and Academic Engagement. Journal of Educational Research, v. 95, n. 6, p. 323-332, 2002.

SIRIAS, D. Using Graphic Organizers to Improve the Teaching of Business Statistics. Journal of Education for Business, v. 78, n. I, p. 33-37, 2002.

SOBRAL, D. T. Motivação do aprendiz de medicina: uso da escala de motivação acadêmica. Psicologia: Teoria e Pesquisa, Brasília, v. I9, n. oI, p. 25-3I, 2003.

VALLERAND, R. J.; BLAIS, M. R.; BRIÉRE, N. M.; PELLETIER, L. G. Construction et validation de l'échelle de motivation en education (EME). Canadian Journal of Behavioural Science, v. 2I, n. 3, p. 323-349, 1989.

VALLERAND, R. J.; PELLETIER, L. G.; BLAIS, M. R.; BRIÉRE, N. M.; SENÈCAL, C.; VALLIÉRES, E. F. The academic motivation scale: a measure of intrinsic, extrinsic, and amotivation in education. Educational and Psychological Measurement, v. 52, n. 4, p. IOO3-IOI7, 1992.

VENDRAMINI, C. M. M. Implicações das atitudes e das habilidades matemáticas na aprendizagem dos conceitos de estatística. 1999. 249 f. Tese (Doutorado em Educação) Universidade Estadual de Campinas, Campinas, 2000.

VENDRAMINI, C. M. M.; SILVA, M.; DIAS; D. Avaliação de atitudes de estudantes de psicologia via modelo de crédito parcial de TRI. Psico-USF, v. I4, n.3, p. 2I-22, 2009. Disponível em: < http://www.scielo.br/scielo.php?pid=SI4I382712009000300005\&script=sci_arttext $>$. Acesso em: ro/04/2010. 
ATITUDE E MOTIVAÇÃO EM RELAÇÃO AO DESEMPENHO ACADÊMICO DE ALUNOS DO CURSO DE

GRADUAÇÃO EM ADMINISTRAÇÃO EM DISCIPLINAS DE ESTATÍSTICA: FORMAÇÃO DE CLUSTERS

VENDRAMINI, C. M. M.; SILVA, C. B.; KATAOKA, V. Y.; CAZORLA, I. M. Validity evidences of the attitudes towards statistics scale SATS: a study with brazilian students. In: Word Statistics Congress, 58., 20II, Dublin. Anais... Dublin, $201 \mathrm{I}$.

Disponível em: < http://isi2orr.congressplanner.eu/pdfs/950I55.pdf>. Acesso em: 20/OI/20I2.

WIBERG, M. Teaching Statistics in Integration with Psychology. Journal of Statistics Education, v. I7, n.I, 2009. Disponível em: < www.amstat.org/publications/ jse/vi7nI/ wiberg.html>. Acesso em: II/07/20II.

WISE, S. L. The development and validation of a scale measuring attitudes toward statistics. Educational and psychological measurement, v. 45, n.2, p. 40I-405, 1985.

WITTER, G.; LOMÔNACO, J. F. Psicologia da aprendizagem. 9.ed. São Paulo: EPU, I984.

WOOLFORD, S.; CLEARY, R. The business of desire and fear. The American Statistical, v. 64, n.I, p. 2I-22, 2010. 


\section{DADOS DOS AUTORES}

GUSTAVO SALOMÃo VIANA`salomao@fearp.usp.br

Mestre em Administração pela FEA/USP

Instituição de vinculação: Faculdade São Luís de Jaboticabal

Jaboticabal/SP - Brasil

Áreas de interesse em pesquisa: Métodos quantitativos e informática.

${ }^{\star}$ Rua Floriano Peixoto, 839 Centro Jaboticabal/SP 14870-370

ADRIANA BACKX NORONHA VIANA backx@usp.br

Livre-docente em Administração pela FEA/USP

Instituição de vinculação atual: Universidade de São Paulo

São Paulo/SP - Brasil

Áreas de interesse em pesquisa: Métodos quantitativos e informática. 